\title{
Perforated Jejunal Diverticulum - A Rare Acute Abdomen Presentation
}

\author{
Manish Verma ${ }^{1}$, Anuj Kumar ${ }^{2}$, Udipt Shringi ${ }^{3}$, Zaheen Khan ${ }^{4}$, M G Vashist ${ }^{5}$ \\ ${ }^{1}$ Associate Professor, Department of Surgery, PGIMS, Rohtak, Haryana, India \\ ${ }^{2}$ Resident, Department of Surgery, PGIMS, Rohtak, Haryana, India \\ ${ }^{3}$ Senior Resident, Department of Surgery, PGIMS, Rohtak, Haryana, India \\ ${ }^{4}$ Resident, Department of Surgery, PGIMS, Rohtak, Haryana, India \\ ${ }^{5}$ Senior Professor, Department of Surgery, PGIMS, Rohtak, Haryana, India
}

\begin{abstract}
Jejunal diverticula are rare and mostly asymptomatic. However, they may lead to chronic non-specific abdominal symptoms such as diarrhea, chronic abdominal pain, bloating or rarely, can present as small bowel perforation. In case of perforation, the recommended treatment is resection and anastomosis. Such a rare case was encountered and managed successfully.
\end{abstract}

Keywords: acute abdomen, jejunal diverticula, small bowl perforation

\section{Introduction}

Intestinal diverticula are mostly present in colon. Jejunal diverticula are rare with an incidence of $0.1-1.5 \%$ [1]. Pathologically, they are pseudodiverticula of pulsion type, resulting from increased intra-luminal pressure and weakening of the bowel wall. These outpouchings contain only mucosa and submucosa. Despite most cases of jejunal diverticulosis remaining completely asymptomatic, complications are reported in 10 to $30 \%$ of patients [2]. These include chronic abdominal pain, malabsorption, hemorrhage, diverticulitis, obstruction, abscess formation and rarely diverticular perforation [3]. Such a rare case of jejunal diverticula presenting as perforation peritonitis is being described.

\section{Case report}

A 65-year-old Indian female was admitted with a 3-days history of worsening abdominal pain associated with nausea. Physical examination revealed tachycardia of 104 beats/min and blood pressure of 110/70mmhg. On clinical examination, there was evidence of generalized abdominal tenderness associated with guarding. Laboratory investigations revealed white cell count of $17 \times 109 / \mathrm{L}$. Rest of the haematological, biochemical and coagulation investigations were within normal limits. Erect plain chest xray showed free air under the diaphragm and abdominal radiograph showed a nonspecific bowel gas pattern. The patient was adequately resuscitated and underwent an exploratory laparotomy. Intraoperative findings revealed multiple jejunal diverticulae with a large perforation of a single jejunal diverticulum without any significant gross contamination (Figure 1).

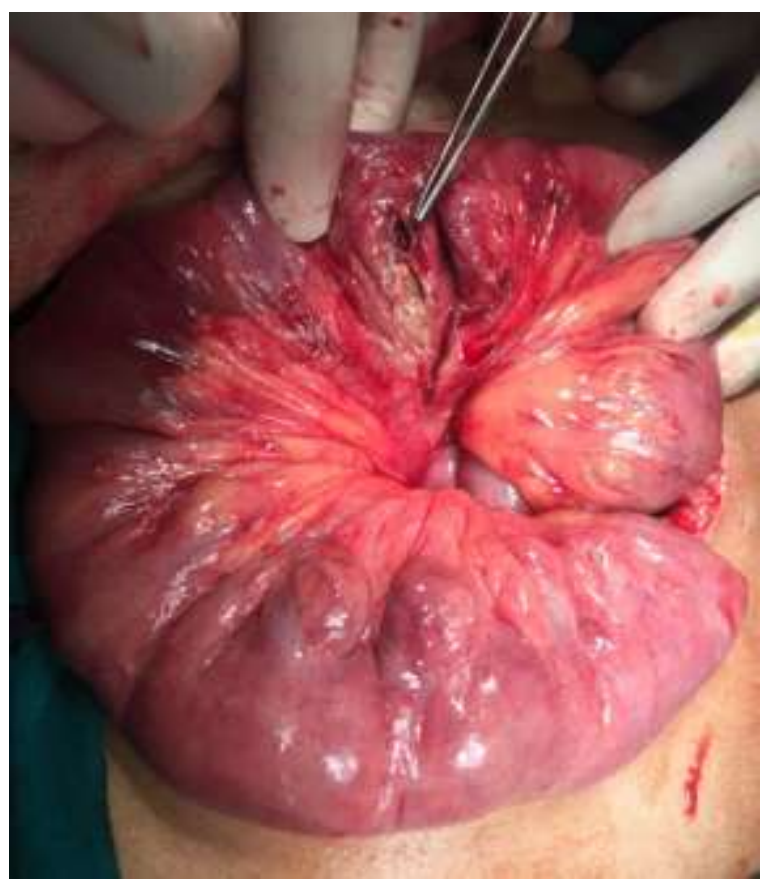

Figure 1: Jejunal diverticula (Intra-operative finding)

The patient underwent a small bowel resection with a primary end-to-end anastomosis and washout of the abdomen. She made an uneventful post-operative recovery and was discharged on day 8. Histopathological examination of the resected specimen confirmed the presence of small bowel diverticular disease.

\section{Discussion}

Jejunal diverticula are the least common type of small bowel diverticula. [1]. The pathologic description of these pseudodiverticula is acquired outpunching of mucosa commonly found on the mesenteric border of the jejunum [4]. Multiple diverticula are seen in $77 \%$ of cases [5]. Clinical diagnosis of diverticular perforation may be difficult

Volume 6 Issue 12, December 2017 


\section{International Journal of Science and Research (IJSR) \\ ISSN (Online): 2319-7064}

Index Copernicus Value (2016): 79.57 | Impact Factor (2015): 6.391

as the symptoms may mimic any other episode of acute abdomen and the diagnosis may easily be confused with other common causes of an acute abdomen such as perforated peptic ulcer and typhoid ulcer perforation. The jejunal diverticula are mostly asymptomatic, however the presenting features are intermittent abdominal pain accompanied by flatulence, diarrhea or constipation in $10 \%$ to $30 \%$ cases [2]. Common acute complications include diverticulitis, bleeding and intestinal obstruction [6]. Abdominal computerized tomography (ACT) has been established as the most valuable imaging technique for Identifying the presence, site and cause of gastrointestinal perforation. ACT with double oral and intravenous contrast may allow the diagnosis of perforated jejunal diverticula, based on the following findings; free intra-peritoneal air; concentrated bubbles of extra luminal air in close proximity to the bowel wall, focal asymmetric wall thickening, edema or thickening of the surrounding fat or fascial planes [7]. However the final diagnosis of diverticula perforation is made intraoperatively and is confirmed on histopathological findings. Asymptomatic diverticular disease found incidentally doesn't require any surgical resection. Treatment is only indicated if complications appear. When a perforation of jejunal diverticula is encountered, resection with anastomosis is required because lesser procedures such as simple closure, excision \& invegination are associated with greater mortality and morbidity rates [8].

\section{Conclusion}

Jejunal diverticula are rare and mostly asymptomatic. However, they may lead to chronic non-specific abdominal symptoms such as diarrhea, chronic abdominal pain, bloating or rarely, can present as small bowel perforation. Clinical presentation is variable. The case described underlines the importance that a surgeon should be aware of such a rare lesion which may mimic as one of the common causes of acute abdomen and must keep it as differential while encountering the cases of acute abdomen.

\section{Other recommendations}

Equalize the length of your columns on the last page. If you are using Word, proceed as follows: Insert/Break/Continuous.

\section{References}

[1] Zager JS, Garbus JE, Shaw JP, Cohen MG, Garber SM: Jejunal diverticulosis: a rare entity with multiple presentations, a series of cases. Dig Surg 2000, 17:6435 .

[2] Akhrass R, Yaffe MB, Fischer C, Ponsky J, Shuck JM: Small-bowel diverticulosis: perceptions and reality. J Am Coll Surg 1997, 184:383-8

[3] Joseph S Butler*, Christopher G Collins and Gerard P McEntee: Perforated jejunal diverticula: a case report. Journal of Medical Case Reports 2010, 4:172

[4] Patel VA, Jefferis H, Spiegelberg B, Iqbal Q, Prabhudesai A, Harris S. Jejunal diverticulosis is not always a silent spectator: a report of 4 cases and reviewof the literature. World J Gastroenterol 2008; 14:5916- 9 .

[5] Lempinen M, Salmela K, Kemppainen E. Jejunal diverticulosis: a potentially dangerous entity. Scand J Gastroenterol 2004; 39: 905-9.

[6] Woods K, Williams E, Melvin W, Sharp K. Acquired jejunoileal diverticulosis and its complications: a review of the literature. Am Surg 2008; 74: 849-54.

[7] Kim SH, Shin SS, Jeong YY, Heo SH, Kim JW, Kang HK. Gastrointestinal tract perforation: MDCT findings according to the perforation sites. Korean J Radiol 2009; 10:63-70.

[8] WH Jennifer, Evers BM. Intestines. In: Townsend CM editor. Sabiston textbook of surgery: The biological basis of modern surgical practice. $20^{\text {th }}$ ed. Vol 2. New Delhi: Elsevier; 2017. p. 1283.

\section{Author Profile}

Manish Verma received the M.S. degrees in General Surgery from PGIMS Rohtak in 2007. During 2007-2009, he stayed in PGIMS Rohtak as Senior Resident in the department of Surgery. For the last 8 years, he is working as consultant in the department of Surgery PGIMS, UHS, Rohtak, Haryana, India.

Volume 6 Issue 12, December 2017 